\title{
A Modified Recovery Procedure to Improve the Accuracy of Stress at Central Area of Bilinear Quadrilateral Element
}

\author{
by Hai Gu*, Student Member Mitsuru Kitamura**, Member
}

\begin{abstract}
Summary
It is found that the accuracy of recovered stress at central area of bilinear quadrilateral element by previous SPR methods ${ }^{1-3)}$ is inferior. Sometimes, the recovered stress is even worse than the raw result of FEA at that area. This may be due to only recovering the nodal stresses and using them to interpolate the stress elsewhere. To overcome this, the recovery procedure is modified by considering additional nodes located at middle point of element edges and the central point of elements and introducing a weighted average procedure to determine the recovered result at some nodes. Numerical analysis of three examples demonstrates the capability of the new procedure to improve the accuracy of recovery at the central area, and the contribution of the weighted average procedure to the stability of recovery at boundary area.
\end{abstract}

\section{Introduction}

There is a difficulty in applying the SPR method, 2,3 ) which has been proved to be one of the most robust and effective $^{4,5)}$ a posteriori error estimators ${ }^{2-10)}$ to nonlinear analysis. That is, the stress can not be recovered by SPR method if the logic of the original version ${ }^{2,3)}$ is completely followed ${ }^{1,6)}$. To overcome this, for bilinear quadrilateral element, in reference [1], the validity and necessity of using the SPR method with $2 \times 2$ integration points as sampling points and recovery polynomial of order two in nonlinear analysis is discussed. Note that only the mesh consists of bilinear quadrilateral elements is considered in this paper.

In both the original SPR method and the method in reference [1], first the patch is defined for each vertex node of the mesh by the union of elements containing that node. After determining an assumed polynomial expansion over the patch for the recovered stress field, the recovered stress at the vertex node and boundary nodes included in the patch is calculated. When the calculation for all patches is finished, the recovered nodal stresses are known. Here, some boundary nodes

* Graduate School, Hiroshima University

** Hiroshima University

Received 10 th July 2000

Read at the Autumn meeting 16, 17 th Nov. 2000) may be included in several patches, and the average of the different recovered values from those patches is used as the finial value of those nodes. Then the nodal stresses along with the same shape functions of FEA are used to calculate the recovered stress elsewhere. That is for each element,

$$
\boldsymbol{\sigma}^{p}=\sum_{i n=1}^{4} N_{i n} \hat{\boldsymbol{\sigma}}^{p}\left(\mathbf{x}_{i n}\right)
$$

where, $\mathbf{x}_{i n}$ is the coordinate vector of the $i n$-th node of the element, $\hat{\boldsymbol{\sigma}}^{p}\left(\mathbf{x}_{i n}\right)$ is the recovered stress of that node, $N_{i n}$ is the bilinear shape function corresponding to the $i n$-th node, and the hat denotes nodal value.

As will be illustrated in numerical examples, by the procedures aforementioned, the accuracy of recovered solution is inferior sometimes even worse than the raw solution of FEA at central area of element. This may be due to that for each patch only nodal stresses are recovered.

In this paper, to enhance the accuracy of recovered solution at the central area, in patch recovery, stress is recovered not only at nodes of the mesh but also at some additional points located at the middle point of element edges and the central point of elements as shown in Fig.1c (denotes these added nodes as "additional nodes" in the following). Then, stress is interpolated by using quadratic Lagrangian shape functions as,

$$
\boldsymbol{\sigma}^{p}=\sum_{i n=1}^{9} \bar{N}_{i n} \overline{\hat{\boldsymbol{\sigma}}}^{p}\left(\mathbf{x}_{i n}\right)
$$


where, the over bar denotes the extended vector to consider the additional nodes, $\bar{N}_{i n}$ is the quadratic Lagrangian shape function corresponding to the in-th component of the nodes series that includes the additional nodes. Note that for each element five additional nodes are added, hence, in Eq.(2) the number of nodes per element is nine.

Just as boundary nodes, some additional nodes may be included in several patches, and different recovered stress at those nodes may be computed from different patch. In both the original SPR method and the method in reference [1], the simple average procedure is implemented to determine the finial value of recovered stress at that kind of nodes. By this way, the recovered values from different patches have same contribution to the finial value. However, in fact, for some patches, the recovered result may have higher accuracy than that of the others. Therefore, it is more reasonable to use some kind of weighted average method and determine the weights according to the quality of recovery.

In this paper, the quality of recovery of a patch is indicated by the difference between the recovered stress obtained in the calculation of this patch and the raw solution of FEA at the integration points in this patch (denoted by Gauss jump of the patch). Similarly, the quality of recovery over an element of the patch is indicated by the Gauss jump of the element. As for the nodes recovered in the patch and needing average, the quality is indicated by the Gauss jump of the elements selected by a way that will be discussed later.

The finial value of the recovered nodal (including the additional nodes) stress is calculated by a weighted average method with the weights determined according to the Gauss jumps. Then the new procedure will be discussed step by step.

\section{The least square problem}

It is value to recall the position of the recovery procedure in the adaptive nonlinear finite element analysis. After the solution of each incremental step is obtained, the discontinuous stress solution of FEA is smoothed by the recovery procedure to a continuous stress solution. Then the error is estimated by the recovery based estimators, and used to guide the adaptive process. In this paper, not the entire adaptive procedure, but the enhancing of the recovery accuracy is the objective. Hence, for simple, the recovery is performed only after finite element analysis is finished.

Obviously the finite element solution is known at the beginning of recovery process. The patch is defined for each vertex node by the union of elements containing that node. Then for each patch, an assumed stress field over the patch represented by a polynomial expansion is determined by assuming a least square fit of the expansion to the finite element solution at a set of sampling points. That is to solve the least square problem shown by Eqs.(3)-(5), 1,3 )

$$
\begin{gathered}
\min F\left(\sigma_{j}^{p}\right)=F\left(\tilde{\sigma}_{j}^{p}\right) \\
F\left(\sigma_{j}^{p}\right)=\sum_{i=1}^{n s \times n e}\left(\sigma_{j}^{p}\left(x_{i}, y_{i}\right)-\sigma_{j}^{h}\left(x_{i}, y_{i}\right)\right)^{2} \\
\sigma_{j}^{p}=\mathbf{P a}
\end{gathered}
$$

where $\mathbf{P}$ contains the appropriate polynomial terms, and in this paper,

and

$$
\mathbf{P}=\left\lfloor\begin{array}{llll}
1 & x & y & x y
\end{array}\right\rfloor
$$

$$
\mathbf{P}=\left\lfloor\begin{array}{llllll}
1 & x & y & x y & x^{2} & y^{2}
\end{array}\right\rfloor
$$

are used; $\mathbf{a}$ is a set of unknown parameters. In this paper, Eulerian formulation is used for plane large deformation problems, $j=1,2,3$ represents the component of Cauchy stress tensor. $\boldsymbol{\sigma}^{h}$ denotes the stress vector obtained from finite element analysis; $\left(x_{i}, y_{i}\right)_{i=1}^{n s \times n e}$ denotes the coordinates of a set of sampling points in the patch on the deformed configuration; $n s$ is the number of sampling points per element, as shown in Fig.1, for the original SPR method $n s$ is 1 , while for the procedure in reference [1], ns is 4 ; ne is the number of elements in the patch.

\section{Patch calculation, computation of the Gauss jump for nodes included in the patch}

After solving Eq.(3) to get the coefficient vector ã the recovered stress at nodes included in the patch is calculated by insertion of appropriate coordinates into Eq.(5). A node is said to be included in a patch when the recovered stress at the node should be calculated in that patch. To improve the accuracy of recovered solution at central area of bilinear quadrilateral element, for each element, five additional nodes located at middle point of element edges and central point of element are considered (see Fig.1c).

In a patch, the vertex node, additional nodes located at central point of elements composing the patch and at the middle point of the edges containing the vertex node (these nodes are enclosed by the dash line in Fig.1c), and nodes (including additional nodes) on the boundary of the mesh will be recovered. Using this way of determining the nodes that should be included in a patch, some nodes may be included in several patches, and different recovered results can be obtained from those patches. Generally, those results do 
not have same accuracy. In this paper, the aforementioned Gauss jump of a node calculated in a patch is employed to indicate the quality of the recovered result of that node in that patch.

In order to calculate the Gauss jump for each node in a patch, the nodes (including the additional nodes) should be classified into 5 types.



(a) Patch with superconvergent points as sampling points(original SPR)



(b) Patch with $2 \times 2$ integration points as sampling points(procedure of Reference [1])



(c) Patch with $2 \times 2$ integration points as sampling points, and with additional nodes(new procedure of this paper)

Fig. 1 Patches of different procedures

1. Vertex node: the assembling node of a patch, and it will be recovered only in that patch, so no average is needed for it.

2. Inter-element additional node (Fig.2a) : the additional node located at middle point of the common edge of two elements. The Gauss jump of this kind of nodes is defined by the sum of the Gauss jumps of those two elements.

3. Central node(Fig.2b): the additional node located at central point of each element. The Gauss jump of the element is considered as the Gauss jump of the node.

4. Boundary additional node (Fig.2c): the additional node located at middle point of boundary edge of element. The Gauss jump of the node is just the Gauss jump of the element.

5. Boundary node (Fig.2d,e): boundary node of the mesh. If in a patch, there is only one element including this node (Fig.2e), then the Gauss jump of this element is considered as the Gauss jump of the node. Otherwise if two elements including the node is included in the patch (Fig.2d), then the larger one between the Gauss jumps of the two elements is considered as the Gauss jump of the node.

The Gauss jump of an element is defined as Eq.(8)

$$
r_{j, i p}^{i e}=\sqrt{\sum_{i g=1}^{n g}\left(\sigma_{j}^{p}\left(\mathbf{x}_{i g}, \text { patchip }\right)-\sigma_{j}^{h}\left(\mathbf{x}_{i g}\right)\right)^{2}}
$$

where,

$$
\begin{aligned}
& r_{j, i p}^{i e}: \text { the Gauss jump of the element } i e \\
& \text { for the stress component } j \text { calculated } \\
& \text { in patch } i p .
\end{aligned}
$$

$n g$ : number of integration points per element.

$\mathbf{x}_{i g}$ : coordinate vector of integration point $i g$.

$\sigma_{j}^{p}\left(\mathbf{x}_{i g}\right.$, patchip $)$ : recovered value of stress component $j$ at integration point $i g$ calculated in patch $i p$.

$\sigma_{j}^{h}\left(\mathbf{x}_{i g}\right)$ : raw result of FEA of stress component $j$ at integration point $i g$.
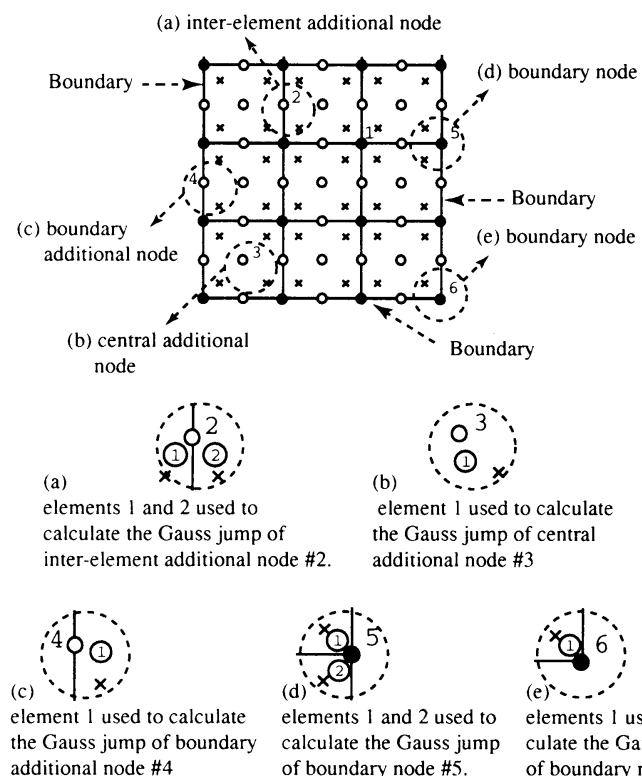

elements 1 and 2 used to calculate the Gauss jump

elements I used to calthe Gauss jump of boundary of boundary node \#5.

culate the Gauss jump of boundary node \#6.

Fig. 2 Classification of nodes

For the five kinds of nodes, the Gauss jump in patch ip can be computed through Eqs.(9)-(13) 
- Vertex node: no average is needed, so,

$$
\hat{r}_{j, i p}^{i n}=0
$$

- Inter-element additional node (Fig.2a)

$$
\hat{r}_{j, i p}^{i n}=\sum_{i e=1}^{2} r_{j, i p}^{i e}
$$

- Central node, boundary additional node (Fig.2b and c)

$$
\hat{r}_{j, i p}^{i n}=r_{j, i p}^{1}
$$

- Boundary node:

1. One element containing the node is included in the patch (Fig.2e)

$$
\hat{r}_{j, i p}^{i n}=r_{j, i p}^{1}
$$

2. Two elements containing the node are included in the patch (Fig.2d)

$$
\hat{r}_{j, i p}^{i n}=\max \left(r_{j, i p}^{1}, r_{j, i p}^{2}\right)
$$

\section{Computation of the recovered nodal stresses and the continuous (recovered) stress field}

After performing the calculation discussed in sections 2 and 3 for all the patches, the final recovered nodal stresses are calculated by a weighted average method shown as Eq.(14).

$$
\hat{\sigma}_{j}^{p}\left(\mathbf{x}_{i n}\right)=\sum_{i p=1}^{n p} w_{j, i p}^{i n} \hat{\sigma}_{j}^{p}\left(\mathbf{x}_{i n}, \text { patchip }\right)
$$

where,

$$
\begin{aligned}
& \hat{\sigma}_{j}^{p}\left(\mathbf{x}_{i n}\right): \text { the final recovered value of the } \\
& \text { stress component } j \text { for node } i n . \\
& \hat{\sigma}_{j}^{p}\left(\mathbf{x}_{i n}, \text { patchip }\right): \text { recovered value of the } \\
& \text { stress component } j \text { for node } i n \text { calcu- } \\
& \text { lated from patch } i p . \\
& n p: \text { number of patches including node } \\
& i n . \\
& w_{j, i p}^{i n}: \text { weight determined by Eq. }(15) \\
& w_{j, i p}^{i n}=\frac{\hat{w}_{j, i p}^{i n}}{\sum_{j p=1}^{n p} \hat{w}_{j, j p}^{i n}} \\
& \hat{w}_{j, i p}^{i n}=\frac{\sum_{j p=1}^{n p} \hat{r}_{j, j p}^{i n}}{\hat{r}_{j, i p}^{i n}}
\end{aligned}
$$

Note that for vertex nodes, no average is needed, so, the recovered stress from the patch assembled by the node is the finial value.

In fact, an assumption is implied in Eqs.(14)-(16). That is the smaller is the Gauss jump of a patch the larger should be the contribution of the recovered result from this patch to the finial value. It is observed numerically that even one very inaccurate recovered solution can decrease the SPR solution and the weighted average method can overcome this drawback. Recalling the patch recovery in SPR method which attempts to achieve a least square fit with respect to the raw result at sampling points, it is reasonable to expect that the weighted average method may benefit the performance of the recovery. Though the weighted average procedure is discussed here only for the new method of this paper, it is easy to be realized that it can also be applied to the original SPR method and the method in reference [1] to determine the recovered stresses of boundary nodes. Then the continuous stress field over the mesh can be obtained by using Eq.(2).

\section{Hyperelastic material}

Hyperelastic materials are employed in this paper in order to apply the original SPR method to recover stress in nonlinear analysis ${ }^{1)}$ and then to compare it with the new procedure. Two kinds of hyperelastic materials are used in numerical examples.

The plane stress incompressible neo-Hookean material is defined by a hyperelastic potential $\phi(\mathbf{C})$ given as,

$$
\phi(\mathbf{C})=\frac{1}{2} \mu(\operatorname{tr} \mathbf{C}-3)
$$

and the corresponding Cauchy stress tensor given as,

$$
\overline{\boldsymbol{\sigma}}=\mu\left(\overline{\mathbf{b}}-j^{2} \overline{\mathbf{I}}\right), j^{2}=\operatorname{det}(\overline{\mathbf{b}})
$$

As for the plane strain compressible neo-Hookean material,

$$
\phi(\mathbf{C})=\frac{1}{2} \mu\left(I_{\mathbf{C}}-3\right)-\mu \ln J+\frac{\lambda}{2}(\ln J)^{2}
$$

and

$$
\boldsymbol{\sigma}=\frac{\mu}{J}(\mathbf{b}-\mathbf{I})+\frac{\lambda}{J}(\ln J) \mathbf{I}
$$

where, $\mathbf{C}=\mathbf{F}^{T} \mathbf{F}$ is the right Cauchy-Green deformation tensor; $\mathbf{b}=\mathbf{F} \mathbf{F}^{T}$ is the Left Cauchy-Green deformation tensor; $\mathbf{F}$ is the deformation gradient tensor; $\mu$ and $\lambda$ are material constants; $J^{2}=\operatorname{det}(\mathbf{C})$ is the third invariant of $\mathbf{C} ; I_{\mathbf{C}}=\mathbf{C}: \mathbf{I}$ is the first invariant of $\mathbf{C}$; I denotes the identity tensor; the overline in Eq.(18) indicates the $2 \times 2$ components of a tensor. 


\section{Numerical investigation}

Three examples as shown in Figs.3-5 are analyzed to testify the performance of the proposed procedure. The plane stress incompressible neo-Hookean material is applied for examples 1 and 2 , while the plane strain compressible neo-Hookean material is used for example 3. For examples 1 and 2, the mathematically selected material properties are: $\rho=1.0, t=0.1, \mu=100.0$ that represent density, thickness and Lame coefficient shown in Eq.(18) respectively. As for example $3, \mu=$ $100.0, \lambda=100.0$ ( see Eq.(19)).

For all the examples, two meshes as shown in Figs.6 and 7 with $8 \times 8$ uniform grid (mesh64) and $16 \times 16$ uniform grid (mesh256) are employed to see the tendency of the variation of the recovery accuracy with the variation of element size. A comparatively fine mesh with $32 \times 32$ uniform grid (mesh 1024) plays the role of reference solution. For each mesh, the stress distribution of the recovered solutions and the raw FEM solution along seven lines as shown in Figs. 6 and 7 are calculated, and for each of those solutions, to facilitate the evaluation of the procedures, a relative error $e_{L}$ is calculated through Eq.(21) which can take care of all stress components. The result is listed in Tables 1-6.

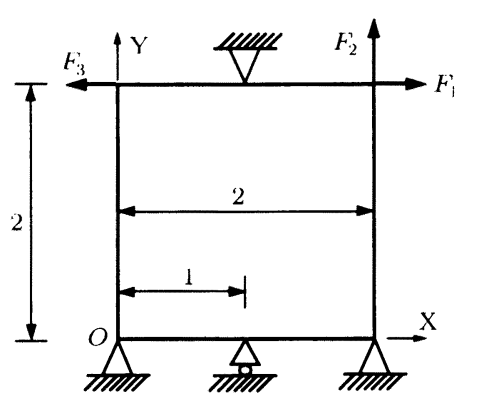

Fig. 3 Example $1, F_{1}=6, F_{2}=18, F_{3}=0.6$

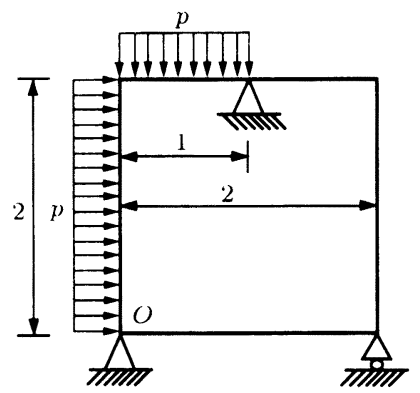

Fig. 4 Example 2, pressure $p=2.0$

$$
\epsilon_{L} \cdots \frac{\left\|\sigma_{x}-\sigma_{x}^{r e f}\right\|_{L}+\left\|\sigma_{y}-\sigma_{y}^{r e f}\right\|_{L}+\left\|\tau_{x y}-\tau_{x y}^{r e f}\right\|_{L}}{\left\|\sigma_{x}^{r e f}\right\|_{L}+\left\|\sigma_{y}^{r e f}\right\|_{L}+\left\|\tau_{x y}^{r e f}\right\|_{L}}
$$

$4 v$

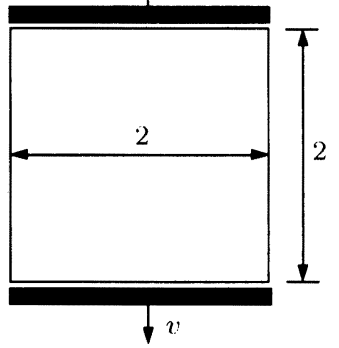

Fig. 5 Example 3, prescribed displacement $v=1.0$

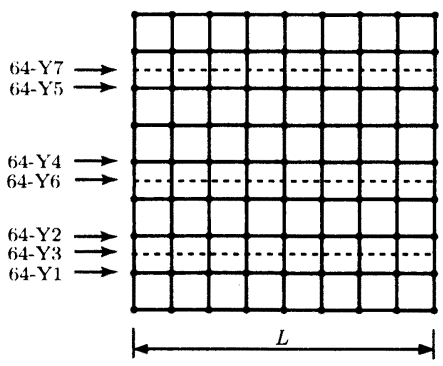

Fig. 6 Mesh64

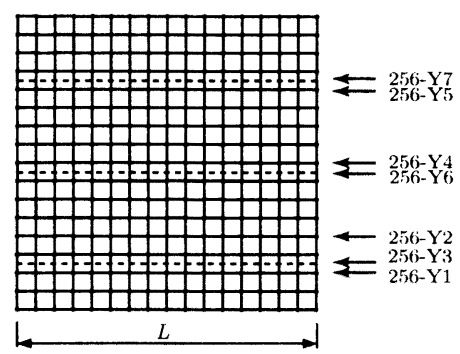

Fig. 7 Mesh256

here,

$$
\begin{aligned}
& \left\|\sigma^{r e f}\right\|_{L}=\int_{L}\left|\sigma^{r e f}\right| d x \\
& \left\|\sigma-\sigma^{r e f}\right\|_{L}=\int_{L}\left|\sigma-\sigma^{r e f}\right| d x
\end{aligned}
$$

The definition of labels used in Tables 1-6 to denote the solution series of different procedures is shown in Table 7.

It can be seen from table 1 that for the stress of mesh64 along line Y3 in example 1 (1-64-Y3), the raw finite element solution has smaller error than that of both the original SPR method (denoted by SAS in this paper) and the procedure in reference [1] (GBS). Similar phenomena (GBS in 1-64-Y7; SAS in 1-256-Y3, 3256-Y3, 2-64-Y3, 2-64-Y7, 2-256-Y3, 3-64-Y3, 3-64-Y6, 3-64-Y7, 3-256-Y6, 3-256-Y7.) can also be observed from the results obtained by previous procedures (SAS and GBS) along lines Y3, Y6 and Y7 that are central line of the elements. That is the reason of concluding at 
Table 1: $e_{L}$ of solution series for example 1 mesh64.

\begin{tabular}{|c|c|c|c|c|c|c|}
\hline \multicolumn{1}{|c}{ GBS } & \multicolumn{1}{c}{ GBW } & \multicolumn{1}{c}{ SAS } & \multicolumn{1}{c|}{ SAW } & \multicolumn{1}{c|}{ GBWA } & FEM \\
\cline { 2 - 7 } $1-64-Y 1$ & 0.527 & 0.5143 & 0.5727 & 0.5422 & 0.4287 & 1.2391 \\
\cline { 2 - 7 } $1-64-Y 2$ & 0.2056 & 0.2031 & 0.2447 & 0.1978 & 0.1862 & 0.5546 \\
\cline { 2 - 7 } $1-64-Y 3$ & 0.4194 & 0.4075 & 0.4648 & 0.4626 & 0.2719 & 0.3857 \\
\cline { 2 - 7 } $1-64-Y 4$ & 0.0858 & 0.0853 & 0.0707 & 0.0698 & 0.0909 & 0.2455 \\
\cline { 2 - 7 } $1-64-Y 5$ & 0.1284 & 0.1033 & 0.1631 & 0.1036 & 0.1028 & 0.2151 \\
\cline { 2 - 7 } $1-64-Y 6$ & 0.116 & 0.1138 & 0.0964 & 0.0972 & 0.095 & 0.1318 \\
\cline { 2 - 7 } $1-64-Y 7$ & 0.4534 & 0.3669 & 0.1711 & 0.1716 & 0.1242 & 0.1812 \\
\hline
\end{tabular}

Table 2: $e_{L}$ of solution series for example 1 mesh256.

\begin{tabular}{|c|c|c|c|c|c|c|}
\hline \multicolumn{1}{|c}{ GBS } & \multicolumn{1}{c}{ GBW } & \multicolumn{1}{c}{ SAS } & \multicolumn{1}{c}{ SAW } & \multicolumn{1}{c|}{ GBWA } & FEM \\
\cline { 2 - 7 } $1-256-Y 1$ & 0.2667 & 0.2246 & 0.9599 & 0.2595 & 0.2053 & 0.5657 \\
\cline { 2 - 7 } $1-256-Y 2$ & 0.1242 & 0.1269 & 0.0624 & 0.063 & 0.096 & 0.4003 \\
\cline { 2 - 7 } $1-256-Y 3$ & 0.2441 & 0.2175 & 0.6237 & 0.2437 & 0.177 & 0.2705 \\
\cline { 2 - 7 } $1-256-Y 4$ & 0.0221 & 0.0228 & 0.0217 & 0.0217 & 0.0224 & 0.1168 \\
\cline { 2 - 7 } $1-256-Y 5$ & 0.0177 & 0.0201 & 0.0262 & 0.0241 & 0.0196 & 0.1075 \\
\cline { 2 - 7 } $1-256-Y 6$ & 0.0253 & 0.0258 & 0.0242 & 0.0242 & 0.0239 & 0.0487 \\
\cline { 2 - 7 } $1-256-Y 7$ & 0.0391 & 0.0329 & 0.0423 & 0.036 & 0.0135 & 0.0428 \\
\hline
\end{tabular}

Table 3: $e_{L}$ of solution series for example 2 mesh64.

\begin{tabular}{|c|c|c|c|c|c|c|}
\hline \multicolumn{1}{|c}{ GBS } & \multicolumn{1}{c}{ GBW } & \multicolumn{2}{c|}{ SAS } & \multicolumn{1}{c|}{ SAW } & \multicolumn{1}{c|}{ GBWA } & \multicolumn{1}{c|}{ FEM } \\
\cline { 2 - 7 } $2-64-Y 1$ & 0.341 & 0.3668 & 0.604 & 0.5916 & 0.2858 & 1.0907 \\
\cline { 2 - 7 } $2-64-Y 2$ & 0.2843 & 0.2674 & 0.3835 & 0.3119 & 0.2302 & 0.8618 \\
\cline { 2 - 7 } $2-64-Y 3$ & 0.3036 & 0.3187 & 0.5636 & 0.5679 & 0.2118 & 0.3905 \\
\cline { 2 - 7 } $2-64-Y 4$ & 0.1169 & 0.1175 & 0.084 & 0.0838 & 0.1193 & 0.3001 \\
\cline { 2 - 7 } $2-64-Y 5$ & 0.1115 & 0.1132 & 0.1259 & 0.1254 & 0.1021 & 0.2799 \\
\cline { 2 - 7 } $2-64-Y 6$ & 0.1557 & 0.1532 & 0.0941 & 0.0931 & 0.1358 & 0.2146 \\
\cline { 2 - 7 } $2-64-Y 7$ & 0.1881 & 0.1886 & 0.2648 & 0.2646 & 0.0922 & 0.23 \\
\hline
\end{tabular}

Table 4: $e_{L}$ of solution series for example 2 mesh256.

\begin{tabular}{|c|c|c|c|c|c|c|}
\hline \multicolumn{1}{|c}{ GBS } & \multicolumn{1}{c}{ GBW } & \multicolumn{1}{c}{ SAS } & \multicolumn{1}{c|}{ SAW } & \multicolumn{1}{c|}{ GBWA } & \multicolumn{1}{c|}{ FEM } \\
\cline { 2 - 7 } $2-256-Y 1$ & 0.1374 & 0.128 & 0.2146 & 0.1808 & 0.0927 & 0.528 \\
\cline { 2 - 7 } $2-256-Y 2$ & 0.1242 & 0.1269 & 0.0624 & 0.063 & 0.096 & 0.4003 \\
\cline { 2 - 7 } $2-256-Y 3$ & 0.1538 & 0.1536 & 0.2511 & 0.2195 & 0.0993 & 0.1866 \\
\cline { 2 - 7 } $2-256-Y 4$ & 0.0348 & 0.0347 & 0.0263 & 0.0263 & 0.0388 & 0.1318 \\
\cline { 2 - 7 } $2-256-Y 5$ & 0.0354 & 0.0356 & 0.0356 & 0.0356 & 0.0308 & 0.1446 \\
\cline { 3 - 7 } $2-256-Y 6$ & 0.0374 & 0.0372 & 0.027 & 0.0268 & 0.0402 & 0.0928 \\
\cline { 2 - 7 } $2-256-Y 7$ & 0.0433 & 0.0434 & 0.0416 & 0.0416 & 0.0275 & 0.0896 \\
\hline
\end{tabular}

the beginning of the paper that the accuracy of the recovered result is inferior sometimes even worse than the raw solution of FEA at central area of bilinear quadrilateral element. Recalling the objective of recovery procedure that is to obtain better result than the original one from FEA, this is obviously an important shortcoming of the pervious procedures. However, by using the method discussed in the former sections (GBWA), better result than the raw FEA solution is obtained in all the cases, which demonstrates the capability of the proposed procedure to overcome the drawback.
Table 5: $e_{L}$ of solution series for example 3 mesh64.

\begin{tabular}{|c|c|c|c|c|c|c|}
\hline \multirow{3}{*}{$3-64-Y 1$} & GBS & \multicolumn{1}{c}{ GBW } & \multicolumn{1}{c}{ SAS } & \multicolumn{1}{c}{ SAW } & \multicolumn{1}{c|}{ GBWA } & \multicolumn{1}{c|}{ FEM } \\
\cline { 2 - 7 } 3-64-Y2 & 0.012 & 0.0115 & 0.0208 & 0.019 & 0.014 & 0.1748 \\
\cline { 2 - 7 } $3-64-Y 3$ & 0.0028 & 0.0027 & 0.0127 & 0.0117 & 0.0052 & 0.1383 \\
\cline { 2 - 7 } 3-64-Y4 & 0.0111 & 0.0102 & 0.0182 & 0.018 & 0.0062 & 0.0121 \\
\cline { 2 - 7 } 3-64-Y5 & 0.0033 & 0.0036 & 0.0055 & 0.0054 & 0.0032 & 0.0944 \\
\cline { 2 - 7 } 3-64-Y6 & 0.0027 & 0.0031 & 0.0073 & 0.0073 & 0.0028 & 0.0034 \\
\cline { 2 - 7 } 3-64-Y7 & 0.019 & 0.0175 & 0.0276 & 0.0276 & 0.01 & 0.02 \\
\hline
\end{tabular}

Table 6: $e_{L}$ of solution series for example 3 mesh256.

\begin{tabular}{|c|c|c|c|c|c|c|}
\hline \multicolumn{1}{|c}{ GBS } & \multicolumn{2}{c}{ GBW } & \multicolumn{1}{c}{ SAS } & \multicolumn{1}{c|}{ SAW } & \multicolumn{1}{c|}{ GBWA } & \multicolumn{1}{c|}{ FEM } \\
\cline { 2 - 7 } $3-256-Y 1$ & 0.0022 & 0.0022 & 0.007 & 0.0059 & 0.0037 & 0.0839 \\
\cline { 2 - 7 } $3-256-Y 2$ & 0.0009 & 0.001 & 0.0027 & 0.0027 & 0.0013 & 0.0684 \\
\cline { 2 - 7 } $3-256-Y 3$ & 0.0032 & 0.003 & 0.0061 & 0.0057 & 0.002 & 0.0058 \\
\cline { 2 - 7 } $3-256-Y 4$ & 0.0008 & 0.0009 & 0.0013 & 0.0013 & 0.0008 & 0.0471 \\
\cline { 2 - 7 } $3-256-Y 5$ & 0.0016 & 0.0017 & 0.0031 & 0.0031 & 0.0016 & 0.0376 \\
\cline { 2 - 7 } $3-256-Y 6$ & 0.0007 & 0.0007 & 0.0018 & 0.0018 & 0.0008 & 0.0014 \\
\cline { 2 - 7 } $3-256-Y 7$ & 0.0042 & 0.0041 & 0.0064 & 0.0064 & 0.0012 & 0.0059 \\
\hline
\end{tabular}

Table 7: Definition of labels in Tables 1-6

\begin{tabular}{|c|c|c|c|c|}
\hline & $\begin{array}{l}\text { Sampling } \\
\text { points }\end{array}$ & $\begin{array}{l}\text { Recovery } \\
\text { function }\end{array}$ & $\begin{array}{l}\text { Average } \\
\text { method }\end{array}$ & $\begin{array}{l}\text { Additional } \\
\text { nodes }\end{array}$ \\
\hline GBS & $2 * 2$ integration points & Eq. (7) & Simple average & No \\
\hline GBW & $2 * 2$ integration points & $\mathrm{Eq} \cdot(7)$ & Weighted average & No \\
\hline SAS & Superconvergent points & Eq. (6) & Simple average & No \\
\hline SAW & Superconvergent points & Eq. $(6)$ & weighted average & No \\
\hline GBWA & $2 \star 2$ integration points & Eq. (7) & Weighted average & Yes \\
\hline FEM & \multicolumn{4}{|c|}{ Finite element solution } \\
\hline $1-64-Y_{1}$ & \multicolumn{4}{|c|}{$\begin{array}{l}\text { Result of mesh64 along line } y 1 \text { in example } 1 . \\
\text { (the others have similar meaning) }\end{array}$} \\
\hline
\end{tabular}

Careful contrast of the errors of solutions along central lines of element ( $Y 3, Y 6, Y 7)$ with those along element edges (Y2, Y4, Y5) shows up an tendency that the raw FEA solution tends to have higher accuracy at central area of element than along element edges while the recovered solutions have the contrary tendency. To illustrate this clearly, the deterioration of recovery accuracy at lines located at central of element is indicated by $\Delta$ calculated through Eqs.(22) and (23), and the result is plotted in Fig.8, in which the values in brackets are the average values of $\Delta$.

$$
\begin{aligned}
& \Delta_{Y 3(Y 6, Y 7)}(\cdot) \\
& =\frac{e_{L}^{Y 3(Y 6, Y 7)}(\cdot)-e_{L}^{Y 2(Y 4, Y 5)}(\cdot)}{e_{L}^{Y 2(Y 4, Y 5)}(\cdot)}
\end{aligned}
$$

an exception is for $256-\mathrm{Y} 3$

$$
\begin{aligned}
& \Delta_{256-Y 3}(\cdot) \\
& =\frac{e_{L}^{256-Y^{3}}(\cdot)-e_{L}^{256-Y 1}(\cdot)}{e_{L}^{256-Y^{1}}(\cdot)}
\end{aligned}
$$






Fig. 8 Deterioration of recovery accuracy at central area of element

From Fig.8, it can be seen that $\triangle$ (FEM) is always negative, which means that the raw FEA solution has higher accuracy at central area of element comparing with that along element edges, $\Delta$ (SAS), $\Delta(\mathrm{SAW}), \Delta(\mathrm{G}$ $B S)$, and $\Delta(\mathrm{GBW})$, contrariwise, are positive in most of the cases. $\Delta$ (GBWA) is negative in half of the cases and positive in the other half while the average value is negative(-0.0103. in fact near zero). Therefore, in general, the accuracy deterioration of solution of GBWA at central area of element is much smaller than that of other recovery procedures, which is obviously an advantage.

The weighted average procedure is also applied to the original SPR method and the method of Reference [1] to determine the final recovered value of some boundary nodes. By comparing SAS with SAW and CBS with GBW, the effectiveness of the weighted average procedure can be investigated. Note that in these. cases, only the recovery at boundary elements will be affected hy average procedure. Hence, in most of the cases, no big difference can be observed between the results of the methods using simple average (SAS and GBS) and that of methods using the weighted average(SAW and (iBW). However, in 1-256-Y1 and 1-256-Y3, much better results are obtained by SAW than by SAS. The solution series in these two cases are plotted in Figs.9 and 10. It is easy to find that the great enhancement achieved by SAW is contributed to the improvement of recovery accuracy at boundary area. Similar but not so significant phenomena can also be observed for some other cases such as 2-64-Y2, 2-256-Y1 and so on. Therefore. the weighted average method may be benefit for the stability of the original SPR procedure at boundary area, and it is particularly effective when the original error is relatively large. Not like for the original SPR method (SAS), the weighted average procedure seems not so necessary for the procedure of Reference 1 ! (GBS), because GBS is more stable at boundary area than SAS.



Fig. 9 Investigation of the capability of the weighted average procedure to enhance recovery accuracy at boundary area by comparing SAS and SAW to reference solution for $1-256-Y 1$

Though is not always the best, the proposed method (GBWA) is still the most possible one to be the best by observing the tables. Moreover, it is more effective to enhance the accuracy of recovery in cases when the original error is relatively large. In fact, with the refining of mesh, the effect of the quality of sampling points on the performance of recovery procedure will be more and more important, no significant improvement can be expected by using more sampling points and higher order recovery functions (which are two features of GBWA), to the contrary, the original SPR method using superconvergent points as sampling points will show its superiority in this situation. Moreover, the error tends to be equilibrated over the whole domain with refining of the mesh. Recalling the discussion in sections 3 and 4 , in this case, the use of weighted average procedure will not be so necessary.Therefore, it may be concluded that the proposed method is particularly fit for the situation when the mesh is relatively coarse.

\section{Conclusion}

The recovery procedure considering additional nodes and using weighted average procedure can improve the accuracy of recovered solution at the central area of bilinear quadrilateral element so as to obtain better solution than the original one of FEM. It is particularly fit for the case where the original error is relatively large, while the original SPR method may be more fit for the 




Fig. 10 Investigation of the capability of the weighted average procedure to enhance recovery accuracy at boundary area by comparing SAS and SAW to reference solution for 1-256-Y3

case where the mesh has been refined to certain degree. Therefore, the procedure GBWA is recommended to be used in the early stage of the adaptive procedure for nonlinear analysis, and the original SPR method to the later stage of the procedure, given that the superconvergent points are available and can be used as sampling points.

The weighted average procedure discussed in this paper can also be applied to previous recovery methods, and it is more likely to improve their performance (enhance the recovery accuracy and robustness) than to deteriorate them slightly sometimes. Certainly, a little more computation cost is needed.

Better solution than the raw FEA solution is purposed to be obtained by recovery procedure. However, hecause of the existence of pollution error ${ }^{7)}$, no guarantee can be made to achieve that goal if local recovery procedure is employed. From numerical investigation of this paper, the proposed method (GBWA) is nearest to that objective, while theoretical study is still needed.

\section{References}

1) M. Kitamura, H. Gu, H. Nobukawa: 'A study of applying the Superconvergent Patch Recovery (SPR) method to large deformation problem', J. Society of Naval Architectures of Japan, 187(2000), pp. 201-208.

2) O.C. Zienkiewicz, J.Z. Zhu: 'The superconvergence patch recovery and a posteriori error esti- mates, part I: the recovery techniques', Int. J. Numer. Meth. Engng., 33. (1992), pp. 13311364.

3) O.C. Zienkiewicz, J.Z. Zhu: 'The superconvergence patch recovery and a posteriori error estimates, part II: error estimates and adaptivity. Int. J. Numer. Meth. Engng., 33, (1992), pp. 1365-1382.

4) I. Babuska. T. Strouboulis: 'Validation of A posteriori Error estimators by numerical approach'. Int. J. Numer. Meth. Engng.. 37, (1994), pp. 1073-1124.

5) J.Z. Zhu: 'A posteriori error estimation - the relationship between different procedures', Comput. Methods Appl. Mech. Engng., 150, (1997). pp. 411-422.

6) B. Boroomand, O.C. Zienkiewicz: 'Recovery procedures in error estimation and adaptivity Part II: adaptivity in nonlinear problems of elastoplasticity behaviour', Comput. Methods Appl. Mech. Engng, 176, (1999), pp.127-146.

7) I. Babuska, T. Strouboulis, S.K. Gangaraj, C.S. Upadhyay: 'Pollution error in the h-version of the finite element method and the local quality of the recovered derivatives', Comput. Methods Appl. Mech. Engng,140(1997), pp.1-37.

8) H. Ohtsubo, M. Kitamura: 'Element by element a posteriori error estimation and improvement of stress solutions for two-dimensional elastic problems', Int. J. Numer. Meth. Engng., 29, (1990), pp. 233-244.

9) H. Ohtsubo, M. Kitamura: 'Element by element a posteriori error estimation of the finite element analysis for three-dimensional elastic problems', Int. J. Numer. Meth. Engng., 33, (1992), pp.1755-1769.

10) M. Ainsworth, J. T. Oden: 'A posteriori error estimation in finite element analysis', Comput. Methods Appl. Mech. Engng., 142, (1997), pp. $1-88$. 